\title{
Évaluation de la production orale en deug scientifique
}

Patrick Doucet

\section{CpenEdition}

Journals

Édition électronique

URL : http://journals.openedition.org/asp/4363

DOI : 10.4000/asp.4363

ISSN : 2108-6354

Éditeur

Groupe d'étude et de recherche en anglais de spécialité

Édition imprimée

Date de publication : 1 mars 1993

Pagination : 85-105

ISSN : 1246-8185

Référence électronique

Patrick Doucet, «Évaluation de la production orale en deug scientifique », ASp [En ligne], 1 | 1993, mis en ligne le 24 avril 2014, consulté le 05 mai 2019. URL : http://journals.openedition.org/asp/4363 ; DOI : $10.4000 /$ asp.4363

Ce document a été généré automatiquement le 5 mai 2019.

Tous droits réservés 


\title{
Évaluation de la production orale en deug scientifique
}

\author{
Patrick Doucet
}

\section{Présentation du public visé}

1 Étudiants du DEUG A (Sciences fondamentales et Sciences expérimentales)

2 Étudiants non volontaires ( 50 heures obligatoires en $2^{\mathrm{e}}$ année)

3 Groupes de TD de 35 à 45 étudiants chacun (poids de l'institution)

4 Attentes de l'institution: une note chiffrée sur 40 (cette note n'est prise en compte que pour les étudiants admissibles)

5 Débouchés : Les secteurs d'emploi ne peuvent pas être définis au niveau du DEUG, si l'on excepte l'Administration (quelques concours «DEUG» possèdent des épreuves scientifiques). Une poursuite d'études est indispensable. Il est à noter que 5 licences seulement sur les 9 qui s'offrent aux titulaires du DEUG A demandent une formation en anglais ${ }^{1}$.

6 À cela il faut ajouter que les étudiants ont une approche essentiellement utilitaire (ils travaillent "pour la note»), qu'ils ont beaucoup de travail par ailleurs, et que dans la majorité des cas, ils ne fournissent aucun travail en dehors des heures de cours.

7 Ces quelques éléments d'information permettront de comprendre pourquoi l'on est en droit de s'interroger sur la motivation des étudiants du DEUG SFA en ce qui concerne l'apprentissage de l'anglais.

\section{Principes méthodologiques pour une évaluation en didactique des langues}

Ginette Barbé ${ }^{2}$ rappelle qu'une méthodologie est une démarche éclairée qui repose sur des principes directeurs s'appuyant sur des connaissances théoriques. Quels sont ces 
principes directeurs? Une conception du langage, une conception de l'apprentissage, une conception socio-pragmatique (que faire de cet apprentissage? quelle utilisation de la langue avons-nous en vue?).

Si l'on s'accorde à prendre pour point de départ que la langue est : un système construit par l'apprenant, un outil, un moyen de communication et d'interaction, nous chercherons donc à mesurer la compétence communicative; nous privilégierons les situations d'interaction, comprenant une négociation du sens.

Si nous avons de l'apprentissage une conception cognitive (exposition/traitement interne/production) (Narcy 1990), il nous faudra insister sur l'aspect individuel, différencié de l'apprentissage.

Dans son ouvrage consacré à l'évaluation dans le contexte d'une approche centrée sur l'apprenant, G. Brindley (1989 : 83) reprend le point de vue de H. Holec (1985) : H. Holec soutient que l'apprenant doit prendre en charge/contrôler son propre apprentissage, et que le rôle de l'enseignant soit davantage gérer des ressources que dispenser le savoir. L'objectif de cette approche est bien entendu l'autonomie de l'apprenant. Le corollaire de cet objectif (que nos étudiants puissent continuer à communiquer et à apprendre à communiquer hors institution) est que nos étudiants deviennent dans la mesure du possible, de bons apprenants de langue.

\section{Quelles sont les caractéristiques du bon apprenant de langue?}

12 Ellis et Sinclair (1983) proposent The good language learner's guide (annexe 1) $)^{3}$. On constate qu'au niveau des stratégies, le bon apprenant de langue doit contrôler et évaluer luimême ses progrès. L'auto-évaluation va donc de pair avec l'objectif fixé à savoir l'autonomie de l'apprenant.

H. Holec fournit les éléments d'une formation à l'auto-évaluation, formation qui comprendrait deux parties, l'une technique et l'autre psychologique. La formation technique à l'auto-évaluation a pour but de permettre aux apprenants de juger leur propre performance, en développant les capacités à observer et auto-contrôler leur usage de la langue, à construire leurs propres critères de jugement, à utiliser leur connaissance descriptive de la langue et à définir leurs propres objectifs ; ce qui nécessite une prise de conscience accrue des phénomènes d'apprentissage (prenant en compte des facteurs tels que la fonction de l'auto-évaluation, la relativité des jugements en ce qui concerne la performance, ainsi que le rôle des erreurs dans les processus d'apprentissage). De plus, $\mathrm{H}$. Holec suggère que l'apprenant soit guidé dans cette démarche. La formation psychologique, selon $\mathrm{H}$. Holec, devrait essentiellement préparer les apprenants à prendre davantage la responsabilité de leur apprentissage, point qui à son avis est un prérequis essentiel pour qu'une auto-évaluation réussie puisse avoir lieu.

Cette formation comprendrait des activités de sensibilisation, destinées à encourager les apprenants à reconsidérer les idées préconçues qu'ils pourraient avoir sur l'apprentissage, ceci afin qu'ils en viennent à changer leur point de vue sur le rôle qu'ils peuvent jouer dans le processus d'apprentissage et en viennent à considérer que l'autoévaluation en est partie intégrante.

16 Dans le cadre de l'enseignement en DEUG scientifique, il est dans l'intérêt des étudiants de développer ces capacités, ce qui leur permettrait de continuer à apprendre de façon 
indépendante les années suivantes, a fortiori si aucune formation en anglais ne leur est proposée (cf. introduction), ou s'ils sont sortis de l'institution.

Cela fait donc partie des objectifs que nous nous fixons. Nous avons des visées extrainstitutionnelles qu'il nous faudra réconcilier avec les exigences de ladite institution.

Il a été dit plus haut que nous chercherions à mesurer la compétence communicative, il convient donc de définir ce terme ${ }^{4}$. L'un des conseils de Meryll Swain ${ }^{5}$ lorsqu'on a à faire face à des problèmes d'ordre pratique est «Start from somewhere » : il faut choisir un point de départ. Il faut construire à partir de connaissances actuelles et d'exemples reconnus ; M. Swain parle de «the gradual and systematic growth in our understanding of the nature of communicative competence » $(1985: 37)$.

19 Le modèle de compétence de communication que nous prendrons pour point de départ sera précisément celui de Canale \& Swain, c'est-à-dire une division en 4 composants majeurs : (1) compétence grammaticale/linguistique, (2) compétence socio-linguistique, (3) compétence discursive, (4) compétence stratégique (ce modèle figure en annexe 2).

\section{Schéma global de Ginette Barbé sur le fonctionnement des pratiques évaluatives}

1. Finalités de l'apprentissage (dépendent du système de valeurs sociales) en rapport pertinent avec

2. Objectifs (définis dans les programmes, au sens large ; ils doivent être mis en question) ; il faut se donner les moyens de vérifier qu'on peut les atteindre

3. Choix de critères

4. Sélection d'informations : recherche des significations du degré d'adéquation entre les indicateurs de performance et le système de référence.

5. Prise de décision

G. Barbé conclut en disant qu'évaluer, c'est « sélectionner des indicateurs et les mettre en rapport avec le système de référence, en vue d'une prise de décision ». Selon G. Barbé, il y a une tendance actuelle qui s'accorde à dire que ce qui guide l'évaluation est avant tout la définition des critères.

21 Ces critères sont toujours abstraits (définis en termes d'être capable de...); ils représentent toujours un choix (il y a toujours plusieurs critères possibles).

Quel est notre choix par rapport à ce schéma? Nous cherchons à développer l'autonomie langagière; nous voulons encourager l'apprenant à communiquer effectivement; nous voulons lui donner les moyens de continuer à apprendre seul. Nous chercherons à favoriser la prise en charge de l'apprentissage par l'apprenant lui-même, par sensibilisation (learner training). 


\section{Quels critères allons-nous choisir pour les étudiants du DEUG?} correspondances qui peuvent exister avec la définition de la compétence communicative que nous avons choisie :

a. Accomplissement de la tâche donnée (met en jeu organisation, cohésion, cohérence -> compétence discursive)

b. Intelligibilité (pertinence pragmatique)

c. Compréhension (interlocuteur et/ou document)

d. d'utilisation de stratégies de communication (compétence stratégique)

e. Débit \& Spontanéité

f. Choix approprié du Registre (compétence socio-linguistique)

g. Absence d'erreurs mécaniques (correction morphologique et syntaxique $\rightarrow$ compétence linguistique).

Il va sans dire que dans le cas d'une évaluation à grande échelle, il nous faudra restreindre le nombre des critères, privilégiant particulièrement ceux qui portent le plus directement sur l'efficacité de la communication. Nous choisirons pour notre expérience les quatre premiers cités (un exemple de typologie de stratégies de communication est donné en annexe 3)

Quel format de test choisir ? (en DEUG scientifique à Poitiers) documentation, s'il y a, et des tâches choisies pour l'élicitation de comportements langagiers communicatifs) doit être par nature: motivant; informatif (certaines des informations contenues dans la documentation doivent être nouvelles pour les apprenants; cela donne un contexte à toutes les tâches que les apprenants auront à accomplir; cela permet de concentrer l'attention des étudiants sur le contenu plutôt que sur la forme, ce qui est une façon de se rapprocher le plus possible d'une situation de communication authentique dans le cadre d'un test. Le test peut avoir une réelle fonction communicative [cf. écart d'information]); intégratif (le test doit se circonscrire autour d'un thème auquel renvoient toutes les informations et activités proposées) et interactif (il y a négociation du sens).

M. Swain (1985) fait également la recommandation suivante : "bias for best ", c'est-à-dire créer des conditions favorables pour optimiser la performance. Notre souci est effectivement de donner l'occasion à l'apprenant de démontrer au mieux ses capacités langagières (de fournir le meilleur « échantillon » à mesurer). Citons Swain :

It is important to minimize the effect of the measurement technique on the test-

taker's performance. (1985: 42)

Conséquemment: le format du test doit permettre l'expression d'un point de vue personnel, une prise d'initiative de la part des apprenants ; le filtre affectif doit être bas (le plus réduit possible).

L'apprenant ne devrait pas se trouver en situation d'infériorité ou sur la défensive: il faudra encourager la coopération et cela jouera sur le type de test que nous choisirons. Il faudra essayer d'éviter que l'apprenant ne se trouve à court de choses à dire (il lui faut, 
comme chacun sait, quelque chose à dire, une raison pour le dire), et nous espérons dans le cadre d'une évaluation qu'il dispose des capacités langagières nécessaires pour l'exprimer ; donnons-lui une tâche à accomplir et assurons-nous que la négociation puisse s'effectuer.

Il faudra également essayer d'éviter que l'apprenant ne soit dérouté par le format du test: l'apprenant doit recevoir une information préalable sur le format du test. L'apprenant ne doit pas être/se sentir " piégé » (ce qui se produit encore trop souvent) : il doit être informé sur ce que l'on cherche à évaluer (une liste de critères/de spécifications doit lui être fournie longtemps à l'avance).

L'information préalable aux étudiants, alliée à la prise en compte des critères susdits dans la pratique évaluative, est la source même de ce que l'on nomme «the washback effect of testing " (note 6,4), un effet de reflux, de retour, qu'a l'évaluation sur les apprenants. Nous parlons entre autres des retombées sur l'attitude de l'apprenant, sur ses stratégies (de production, mais aussi, du moins l'espérons-nous, d'apprentissage) et des retombées sur les formations ultérieures ;

31 Lorsqu'il mentionne cet "effet de reflux », A. Davies $(1990)^{6}$ soutient que le destin d'un test normatif est de devenir le test sommatif de formations subséquentes, par le fait même de la tendance des enseignants à orienter leur programme d'enseignement en fonction du test à venir.

Citons Davies :

However, the reality is that testing is always used in teaching, in the sense that much teaching is related to the testing that is demanded of its students. In other words testing always has a "washback" influence and it is foolish to pretend that it does not happen. (1990:24)

En corollaire, si l'on n'évalue pas la langue orale, elle ne sera pas enseignée. Le conseil d'Alan Davies est le suivant "given the inevitability of washback the sensible thing to do is to accept it and make sure [...] that it provides for satisfactory teaching content and method " (ibid. : 24-25).

Citons encore Jones (in Lee et alii $1985: 79)$ : « We cannot afford to ignore oral testing, if for no other reason than the backwash effect ».

Dans le cadre d'une évaluation critériée, nous devons nous référer à des grilles de niveaux ; nous prendrons comme point de départ les grilles de niveaux de l'UPLEGESS'.

Il semble cohérent avec nos choix de tester nos étudiants en binômes.

\section{Quelles tâches allons-nous proposer à nos étudiants lors des tests ?}

Il s'agira de deux types distincts d'activités ${ }^{8}$ : tout d'abord des activités comprenant un écart d'information entre les intervenants (par exemple en DEUG scientifique, des activités mettant en jeu des descriptions, des comparaisons de documents - repérage dans un espace à deux, à trois dimensions : images ; figures ; blocs de construction...), puis des activités reposant sur une différence d'opinions entre les intervenants (exercices de classement ; éventuellement exercices de résolution de problème...).

Ce format est adaptable à une approche fonctionnelle de l'enseignement des langues: dans la typologie de Van Ek (annexe 4), on reconnaîtra «imparting and seeking factual information, getting things done, expressing and finding out intellectual attitudes ». 


\section{Tableau 1}

\begin{tabular}{|c|c|c|c|}
\hline Critère & $\begin{array}{l}\text { Étudiant A } \\
\text { Nom }\end{array}$ & $\begin{array}{l}\text { Étudiant B } \\
\text { Nom }\end{array}$ & Degré de certitude \\
\hline Achèvement tâche & $\begin{array}{l}2 \\
1 \\
0\end{array}$ & $\begin{array}{l}2 \\
1 \\
0\end{array}$ & $\begin{array}{l}\text { A } 0123 \\
\text { B } 0123\end{array}$ \\
\hline Intelligibilité & $\begin{array}{l}2 \\
1 \\
0\end{array}$ & $\begin{array}{l}2 \\
1 \\
0\end{array}$ & $\begin{array}{l}\text { A } 0123 \\
\text { В } 0123\end{array}$ \\
\hline Utilisation de stratégies de communication & $\begin{array}{l}\text { occasionnelle } \\
2 \\
1 \\
0 \\
\text { systématique }\end{array}$ & $\begin{array}{l}\text { occasionnelle } \\
2 \\
1 \\
0 \\
\text { systématique }\end{array}$ & $\begin{array}{l}\text { A } 0123 \\
\text { B } 0123\end{array}$ \\
\hline $\begin{array}{l}\text { Compréhension information } \\
\text { (interlocuteur/doc) }\end{array}$ & $\begin{array}{l}2 \\
1 \\
0\end{array}$ & $\begin{array}{l}2 \\
1 \\
0\end{array}$ & $\begin{array}{l}\text { A } 0123 \\
\text { В } 0123\end{array}$ \\
\hline
\end{tabular}

On remarquera que dans le cadre de l'expérience, il sera demandé aux examinateurs d'indiquer à quel degré de précision ils pensent avoir évalué les étudiants, car notre souci sera d'établir la fiabilité d'un tel test.

Il nous faudra organiser régulièrement des réunions d'harmonisation entre les examinateurs (tester training: Morrison and Lee 1985 ; Nic Underhill 1987). L'enregistrement des étudiants devrait permettre des retours en arrière et une analyse plus approfondie de l'interaction orale. Peut-être au cours de l'expérience serons-nous amenés à repréciser, à détailler plus avant, certains éléments de nos grilles de niveaux. En outre, il semblerait que le test oral en binôme soit plus fiable que l'entretien classique : en effet l'examinateur peut se consacrer pleinement à l'évaluation.

Il reste à s'interroger sur la validité de ce type de test: nous l'avons conçu pour qu'il reflète de façon appropriée la théorie de l'acquisition/apprentissage mise en œuvre, mais il conviendra de le vérifier par l'expérience. Il faudra également vérifier que l'échantillon produit est satisfaisant: permet-il de rendre compte effectivement du niveau de 
performance des étudiants? (Une précaution utile nous semble être de vérifier par ailleurs la compréhension des étudiants, par des tests de lecture et d'écoute). En ce qui concerne la crédibilité du test, il règne une controverse à ce sujet : Davies (90) pense que s'il y a conflit avec d'autres types de validité, ce type doit être le premier à abandonner ; Stevenson (1985) met en cause son existence même. D'autres (Morrow 1979 ; Swain 1985 ; Underhill 1987) pensent que cette validité est fondamentale car elle conditionne la réponse de l'apprenant: elle est susceptible de susciter la meilleure performance de la part de ce dernier (c'est avant tout une personne et nous avons besoin de son adhésion.) Underhill (1987) se prononce en faveur d'une approche humaine de l'évaluation. Concluons pour l'instant en rappelant que la combinaison des validités renforce la validité de l'ensemble (Davies 1990).

En ce qui concerne la faisabilité du test, nous pensons organiser comme nous l'avons dit plus haut, une double correction par échanges de cassettes audio entre les enseignants intervenant en DEUG. Rappelons que dans la mesure où nous privilégions la production orale aux dépens de la production écrite, le temps habituellement passé à corriger des copies sera agréablement consacré à écouter ces cassettes ; il n'y aura donc pas surcharge de travail pour les enseignants.

Comme il est pour l'instant impossible, institutionnellement parlant, d'évacuer le problème de la notation, nous proposons une notation sur échelle réduite (tout en essayant de convaincre l'institution du bien-fondé d'une approche critériée menant à l'établissement d'un profil). La notation se fera par transformation indirecte, à partir des grilles de niveaux. La note attribuée dépendra d'une négociation entre les examinateurs.

En conclusion, la pratique évaluative que nous proposons en DEUG scientifique comprendra une formation à l'auto-évaluation en cours d'année et un ou plusieurs tests interactifs en binômes. Nous nous proposons de détourner une évaluation normative à des fins formatives.

Ce que l'on pourrait appeler «best test » dans la situation spécifique où nous nous trouvons permet/suscite/encourage une prise de conscience de l'apprenant, en ce qui concerne : l'apprentissage des langues, et partant son propre apprentissage ; les qualités/ caractéristiques qui favorisent cet apprentissage (dans le cadre théorique défini plus haut); les stratégies d'apprentissage qu'il conviendra de développer pour optimiser sa performance. Ce «best test » fera pour nous office de «catalyseur»; il pourra y avoir émergence de besoins nouveaux de formation.

49 Il nous reste bien sûr à vérifier nos hypothèses, à tenir compte de la réaction des apprenants pendant/après le test. Nous serons peut être amenés à réviser notre approche en fonction des résultats. Il nous reste aussi à mesurer l'effet d'un entraînement méthodologique. Des expériences seront mises en place dans les mois à venir.

Voici pour terminer la définition que Nic Underhill donne de «best test » :

This is the test most suitable for the particular situation in which it will be used. Although every test procedure has its own advantages and disadvantages, it is only a good test or a bad test in a particular context [...]. Because the best test is context specific, it can only be designed for, and produced in that context, and no amount of expert authority will make the best test in one place automatically the best test in another." (1987:9)

51 Nous espérons fournir une réponse adaptée à une situation particulière. Nous pensons cependant que les formules proposées sont largement adaptables à d'autres cadres institutionnels. 


\section{BIBLIOGRAPHIE}

Brindley, G. 1989. Assessing Achievement in the Learner-Centered Curriculum. Sydney: National Centre for English Language Teaching and Research, Macquarie University.

Davies, A. 1990. Principles of Language Testing. Applied Language Studies Cambridge : Blackwell.

Jones, R. L. 1985. « Some basic considerations in testing oral proficiency ». In Lee, Y. P. et alii (dir.), New Directions in Language Testing. Oxford : Pergamon Institute of English, 77-84.

Klippel, F. 1984. Keep Talking. Cambridge : Cambridge University Press.

Lee, Y. P. et alii (dir.). 1985. New Directions in Language Testing. Oxford : Pergamon Institute of English.

Morrison, D. M. \& Lee, N. 1985. « Simulating an academic tutorial: A test validation study ». In Lee, Y. P. et alii (dir.), New Directions in Language Testing. Oxford : Pergamon Institute of English, $85-92$.

Narcy, J.-P. 1990. Apprendre une langue étrangère. Paris : Éditions d'organisation.

Stevenson, D.K. 1985. « Pop validity and performance testing ». In Lee, Y. P. et alii (dir.), New Directions in Language Testing. Oxford : Pergamon Institute of English, 111-118.

Swain M. 1985. « Large-scale communicative language testing: A case study ». In In Lee, Y. P. et alii (dir.), New Directions in Language Testing. Oxford : Pergamon Institute of English, 35-46.

Underhill, N. 1987. Testing Spoken Language. Cambridge : Cambridge University Press.

\section{ANNEXES}

\section{Annexe 1. The good language learner's guide}

\section{Personality}

GLLs actively involve themselves in the language learning task.

GLLs are willing to experiment with new learning strategies.

GLLs don't mind taking risks and experimenting with language.

\section{Attitudes}

GLLs realise that language works as an organised system.

GLLs realise that language in a means of communication and interaction.

GLLs realise that it takes a lot of hard work and time to learn a language and this does not happen only in a classroom.

\section{Strategies}

GLLs monitor and evaluate their progress.

GLLs vary learning activities and choose activities that are interesting and meaningful to them.

GLLs learn a little/often and practise regularly.

GLLs organise their learning programme and materials. 
GLLs have certain sub-goals as marks of progress.

Source : Carry on Learning: A Guide Towards Learner Autonomy, The Council of Europe, 1983.

\section{Annexe 2. Compétence communicative (Swain 1983, 1985)}

1. Compétence grammaticale : (préférons le terme linguistique, cf. lexique) degré de maitrise par l'apprenant du code linguistique, y compris vocabulaire, prononciation, orthographe, construction de mots et de phrases essentiel si objectifs élevés. (connaissances déclaratives et procédurales : comprendre et exprimer avec précision le sens littéral des énoncés).

2. Compétence sociolinguistique : choix approprié de formes grammaticales en fonction du contexte sociolinguistique, pour faire passer des fonctions de communication spécifiques (persuader, décrire, raconter, ordonner...).

Ce choix dépend du sujet, du rôle/du statut des participants, du cadre dans lequel la communication a lieu (des objectifs de l'interaction) $=>$ attitude à adopter/choix de style registre.

Compréhension et expression correctes d'énoncés dans différents contextes sociolinguistiques. Dans ce cas, le sens et la forme des énoncés sont adéquats.

3. Compétence discursive : capacité à combiner des idées pour obtenir cohésion de la forme et cohérence de la pensée. Elle permet de donner une unité à l'expression dans différents genres (narration, dissertation, compte-rendu scientifique, lettre d'affaires...).

Cohésion : façon dont les énoncés sont liés structurellement pour faciliter l'interprétation d'un texte (utilisation de mots de liaison, de pronoms, de synonymes, d'ellipses, de structures parallèles... >> compréhension logique et chronologique d'un texte).

Cohérence : relation entre les différents sens d'un texte, qu'ils soient littéraux, qu'ils aient une fonction sociale ou communicative.

4. Compétence stratégique : utilisation de stratégies de communication pour compenser les manques/vides dans la connaissance qu'a la personne du code (pour renforcer l'efficacité de la communication) pour compenser toute autre rupture/interruption de la communication (pannes de communication dues à des facteurs contraignants ou à une compétence insuffisante dans l'un ou l'autre des composants de la compétence de communication). Plus sa compétence de communication augmente et moins on aura besoin de faire appel à sa compétence stratégique (même si celle-ci opère à tous les niveaux)

Annexe 3. Typology of communication strategies (source : Tarone $1980: 429$ )

Paraphrase

Approximation: Use of a single target language vocabulary item or structure, which the leaner knows is not correct, but which shares enough semantic features in common with the desired item to satisfy the speaker (e.g.",pipe" for "waterpipe").

Word coinage: The learner makes up a new word in order to communicate a desired concept (e.g., "airball" for "balloon").

Circumlocution: The leaner describes the characteristics or elements of the object or action instead of using the appropriate TL structure ("She is, uh, smoking something. I don't know what's its name. That's, uh, Persian, and we use in Turkey, a lot of').

Transfer

Literal translation: The learner translates word for word from the native language (e.g., 
"He invites him to drink" for "They toast one another").

Language switch: The learner uses the NL term without bothering to translate (e.g., "ballon" for "balloon" or "tirtil" for "caterpillar").

Appeal for assistance: The learner asks for the correct term or structure (e.g., "What is this?").

Mime: The learner uses nonverbal strategies in place of a meaning structure (e.g., clapping one's hand to illustrate applause).

\section{Avoidance}

Topic avoidance: Occurs when the learner simply does not talk about concepts for which the vocabulary or other meaning structure is not known.

Message abandonment: Occurs when the learner begins to talk about a concept but is unable to continue due to lack of meaning structure, and stops in mid-utterance.

\section{Annexe 4}

Van Ek (1980) distinguishes six main functions of communication:

Imparting and seeking factual information (identifying, reporting, correcting, asking)

Expressing and finding out intellectual attitudes (expressing and inquiring about agreement and disagreement, accepting or declining an offer or invitation, etc.)

Expressing and finding out emotional attitudes (pleasure or displeasure, surprise, hope, intentions, etc.)

Expressing and finding out moral attitudes (apologizing, expressing approval or disapproval, etc.)

Getting things done (suasion) (suggesting a course of action, advising, warning)

Socializing (greeting and leaving people, attracting attention, proposing a toast).

\section{NOTES}

1. La grande majorité des étudiants du DEUG n'a pas accès aux MST ou à l'ESIP (École Supérieure d'Ingénieurs de Poitiers).

2. Je reprendrai ici l'essentiel des propos tenus par Ginette Barbé, psycholinguiste, lors du colloque organisé le 11 octobre 1991 par le British Council, UCLES, et le Bell Educational Trust, sur le thème «L'évaluation de l'apprentissage des langues étrangères dans les Grandes Écoles ».

3. Tableau reproduit p. 121 du livre de J.-P. Narcy (1990). Cf. annexe 1.

4. Ceci demande une certaine prudence : Larsen-Freeman et Long (1991) précisent que l'analyse du concept de compétence de communication est tout aussi insaisissable que l'était celui de " proficiency». Ils précisent en outre que même si l'on arrivait à se mettre d'accord sur les différents composants de la compétence de communication, il reste encore à savoir comment mesurer ces différents composants si l'on veut identifier les processus d'acquisition.

5. Merrill Swain (in Lee et alii 1985) énonce quatre principes généraux particulièrement pertinents lorsqu'il s'agit de faire face à des problèmes d'ordre pratique : (1) Start from somewhere

(2) Concentrate on content (3) bias for best (4) work for washback. Ces principes seront repris au long du présent article.

6. Argument déjà mis en avant dans son article « Follow my leader: Is that what language tests do? » (in Lee et alii 1985 : 3-14).

7. Livre blanc sur l'évaluation des compétences en langues en fin de cursus, décembre 1988, coordination par J.-P. Narcy. 
8. Deux ouvrages sont à citer pour des exemples de tâches communicatives et de techniques d'élicitation : Keep Talking de Friederike Klippel (1984) et Testing Spoken Language de Nic Underhill (1987).

\section{AUTEUR}

\section{PATRICK DOUCET}

Faculté des Sciences fondamentales et appliquées, Université de Poitiers. Patrick.Doucet@univpoitiers.fr. 